\title{
Monotop signature from a fermionic top partner
}

\author{
Dorival Gonçalves, ${ }^{1}$ Kyoungchul Kong, ${ }^{2}$ Kazuki Sakurai, ${ }^{3}$ and Michihisa Takeuchi ${ }^{4}$ \\ ${ }^{1}$ PITT PACC, Department of Physics and Astronomy, University of Pittsburgh, \\ 3941 O'Hara Street, Pittsburgh, PA 15260, USA \\ ${ }^{2}$ Department of Physics and Astronomy, University of Kansas, Lawrence, Kansas 66045, USA \\ ${ }^{3}$ Institute of Theoretical Physics, Faculty of Physics, University of Warsaw, \\ ulica Pasteura 5, PL-02-093 Warsaw, Poland \\ ${ }^{4}$ Kavli IPMU (WPI), UTIAS, University of Tokyo, Kashiwa 277-8583, Chiba, Japan
}

(Received 13 November 2017; published 8 January 2018)

\begin{abstract}
We investigate monotop signatures arising from phenomenological models of fermionic top partners, which are degenerate in mass and decay into a bosonic dark matter candidate, either spin 0 or spin 1 . Such a model provides a monotop signature as a smoking gun, while conventional searches with $t \bar{t}+$ missing transverse momentum are limited. Two such scenarios, (i) a phenomenological third generation extradimensional model with excited top and electroweak sectors, and (ii) a model where only a top partner and a dark matter particle are added to the standard model, are studied in the degenerate mass regime. We find that in the case of extra dimension a number of different processes give rise to effectively the same monotop final state, and a great gain can be obtained in the sensitivity for this channel. We show that the monotop search can explore top-partner masses up to 630 and $300 \mathrm{GeV}$ for the third generation extradimensional model and the minimal fermionic top-partner model, respectively, at the high luminosity LHC.
\end{abstract}

DOI: $10.1103 /$ PhysRevD.97.015002

\section{INTRODUCTION}

Monotop searches have been proposed in a context of supersymmetry (SUSY) especially when the superpartner (stop, $\tilde{t}$ ) of the standard model (SM) top quark $(t)$ is degenerate with (Higgsino-like) neutralinos $\left(\tilde{h}^{0}\right)[1-3] .{ }^{1}$ In such a scenario, $\tilde{t}$ effectively behaves as an invisible particle since its decay products are too soft to be detected, and the sensitivity is therefore deteriorated for the standard $p p \rightarrow \tilde{t}^{*}$ channel $[15,16]$. It has been shown that in this regime the $p p \rightarrow \tilde{t} t \tilde{h}^{0}$ channel can have a measurable production rate due to the large top Yukawa coupling, leading to a characteristic monotop $+E_{T}$ final state, where both $\tilde{t}$ and $\tilde{h}^{0}$ are effectively invisible. Differently than the usual monojet signatures exploiting hard QCD initial state radiation and therefore providing very little information on the produced particles, the monotop signature allows a direct probe of the stop and neutralino sectors [1]. This means that the $\tilde{t} t \tilde{h}^{0}$ channel is complementary to the monojet channel or even essential in exploring the SUSY parameter space. While such

\footnotetext{
${ }^{1}$ The monotop signatures arising from $\tilde{t} \tilde{\chi}^{-}$production in nondegenerate SUSY spectra are discussed in $[4,5]$. Traditionally monotop is often studied in the context of flavor models [6-14].

Published by the American Physical Society under the terms of the Creative Commons Attribution 4.0 International license. Further distribution of this work must maintain attribution to the author(s) and the published article's title, journal citation, and DOI. Funded by SCOAP ${ }^{3}$.
}

a supersymmetric signature is very well motivated theoretically in terms of naturalness [17-35], it is desirable to phenomenologically expand the scope of current monotop studies including different spin scenarios. As supersymmetry provides a spin-0 top partner together with a spin- $1 / 2$ dark matter (DM) candidate, we would like to extend the search to the case with a spin-1/2 top-partner, which decays into either spin- 0 or spin- 1 DM candidate. As in the supersymmetric case, we assume the degeneracy between the fermionic toppartner and bosonic DM candidates.

For the spin-0 top-partner case, "natural SUSY" provides a well-motivated example for the degenerate spectrum. Similarly, for the spin-1/2 top-partner case, such a compressed spectrum is naturally realized in models with extra dimensions. A good benchmark model for the purpose of our analysis is universal extra dimensions (UED), where all SM particles propagate in the bulk of flat extra dimensions, and the mass spectrum of Kaluza-Klein (KK) particles is degenerate [36]. This degeneracy is broken due to electroweak symmetry breaking, bulk and boundary term corrections from the renormalization group running between the cutoff scale and the electroweak scale [37-41]. Nevertheless, the overall mass spectrum is much narrower than that for conventional supersymmetry. This observation strongly encourages revisiting monotop production in the context of extra dimensions.

UED with a particular mass spectrum derived in $[38,39]$ is called minimal universal extra dimensions and has been extensively studied in the literature [37-50]. Recently, it has 
been revisited with the 8 and $13 \mathrm{TeV}$ LHC data with the conventional cascade decays [43,44], and the estimated lower bound on the inverse radius is found to be around $R^{-1} \gtrsim$ $1.4 \mathrm{TeV}$ with some variation in the cutoff scale $(\Lambda)$. For the simplified model with a fermonic top-partner $T$, the lower bound on the mass with $13 \mathrm{fb}^{-1}$ at $13 \mathrm{TeV}$ is estimated as $m_{T} \gtrsim 1.1 \mathrm{TeV}$ for $m_{\chi} \lesssim 450 \mathrm{GeV}$ [51]. These searches with jets, leptons, and missing transverse momentum are promising in general, but their sensitivity gets poor for smaller mass splitting. On the other hand, the monojet channel becomes more sensitive for compressed spectra, which is expected for a low value of $\Lambda R$. This point is examined in Ref. [42], and the authors find that monojet searches result in the current bound $R^{-1} \gtrsim 1.1 \mathrm{TeV}(\sim 1.2 \mathrm{TeV}$ and $\sim 1.3 \mathrm{TeV}$ for the masses of KK quark and KK gluon, respectively) for $\Lambda R \lesssim 5$ with $3.2 \mathrm{fb}^{-1}$ of data at the $13 \mathrm{TeV}$ LHC, which is comparable to the monojet exclusion limits on the masses of squarks ( $\gtrsim 0.8 \mathrm{TeV})$ and gluino $(\gtrsim 1 \mathrm{TeV})$ in supersymmetry $[52,53]$. Since the monotop signals arise from the third generation of KK quarks, we study the monojet channel with the corresponding particle content in our study. This should be compared to Ref. [42], where the entire KK spectrum is included in the analysis.

Although collider phenomenology of extradimensional models has been examined extensively for many years, its monotop signature has not been pursued yet. Therefore, our study is worthwhile and provides useful information concerning SUSY and UED searches. Moreover, in a particular case where only KK tops and KK dark matter candidates are considered without additional KK particles, our analysis is more generally applicable beyond extradimensional models and our results would be still valid in a generic model with fermionic top partners and a dark matter candidate. Hence, although, in this paper, we refer to the fermionic top partner as KK top [denoted by $t^{(1)}$ or $T^{(1)}$, depending on their $S U(2)_{W}$ charge], and the bosonic DM candidate as KK photon $\left(\gamma_{\mu}^{(1)}\right)$ for spin 1 or KK Higgs boson $\left(h^{(1)}\right.$ or $\chi^{(1)}$, depending on their $C P$ property) for spin 0 , our results can be more general. We consider KK numberconserving interactions in our study and therefore all interactions are fixed by the SM ones. Masses of the new particles are treated as free parameters, as in nonminimal UED [40], which we fix following the previous SUSY studies for comparison [1,2]. We also take SUSY decay chains used in the previous study to guarantee an appropriate comparison against the existing results. All SUSY particles are replaced with the corresponding KK partners with different spins.

This paper is organized as follows. In Sec. II, we define two benchmark scenarios that are addressed in our study and describe the top-partner interactions that are relevant to the monotop signature. In Sec. III, we present the result of our numerical study based on Monte Carlo simulations and derive the corresponding LHC bounds. Finally, a summary of our main findings is given in Sec. IV.

\section{RELEVANT INTERACTIONS FOR FERMIONIC TOP PARTNER}

We consider two different scenarios in our monotop study.

(i) A phenomenological third generation extradimensional model, which consists of the top-partner sector $\left[S U(2)_{W}\right.$ singlet $\mathrm{KK}$ top $t^{(1)}$, and third generation $S U(2)_{W}$ doublet, $\left.\left(T^{(1)}, B^{(1)}\right)\right]$ as well as the full KK Higgs and KK electroweak gauge boson spectrum. Such a scenario may be realized in nonminimal UED models [40,54-58].

(ii) A minimal scenario with one fermionic top-partner $t^{(1)}$ and bosonic DM candidate $h^{(1)}$ (spin 0) or $\gamma_{\mu}^{(1)}$ (spin 1), as to stop plus neutralino corresponding to the simplified model in SUSY.

Following particle content and interactions as in UED, we assume the lightest KK particle (LKP) to be electrically neutral and colorless, so as to be the dark matter candidate. As long as the LKP is stable and invisible within the detectors, further specification of the LKP is not important since decays of KK particles are not visible due to the mass degeneracy among them.

The KK photon $\gamma_{\mu}^{(1)}$ is essentially the KK hypercharge gauge boson, $\gamma_{\mu}^{(1)} \approx B_{\mu}^{(1)}$, since the Weinberg angle at KK level is small, $\theta_{W}^{(n)} \ll 1$. Similarly the KK $Z$ consists of mostly neutral component of $S U(2)_{W}$ KK gauge boson, $Z_{\mu}^{(1)} \approx W_{\mu}^{(1) 3}$. This is analogous to the case of pure bino $\tilde{b}$ and zino $\tilde{z}$ in SUSY. $W_{\mu}^{(1) \pm}$ and $H^{(1) \pm}$ are the charged KK $W$ and KK Higgs bosons. We denote $C P$ even and $C P$ odd Higgs bosons as $h^{(1)}$ and $\chi^{(1)}$, respectively. The SM top quark and KK top quarks form the following Dirac fermions:

$$
\begin{aligned}
& t=\left(\begin{array}{c}
T_{L} \\
t_{R}
\end{array}\right), \quad \text { SM top quark, } \\
& T^{(1)}=\left(\begin{array}{c}
T_{L}^{(1)} \\
T_{R}^{(1)}
\end{array}\right), \quad S U(2)_{W} \text { doublet KK top, } \\
& t^{(1)}=\left(\begin{array}{c}
t_{L}^{(1)} \\
t_{R}^{(1)}
\end{array}\right), \quad S U(2)_{W} \text { singlet } \mathrm{KK} \text { top. }
\end{aligned}
$$

Often $t^{(1)}\left(T^{(1)}\right)$ is called the right-handed (left-handed) KK top. However, it is really a vectorlike quark. The handedness refers to the chirality of the SM fermion of its origin, i.e., $t^{(1)}$ is KK partner of the right-handed SM top $t_{R}$ and $T^{(1)}$ is the KK partner of the left-handed SM top $T_{L}$. The relevant interactions involving the SM top quark and the $\mathrm{KK}$ electroweak gauge bosons are

$$
g_{1} \frac{Y_{R}}{2} \bar{t}^{(1)} \gamma^{\mu} P_{R} t \gamma_{\mu}^{(1)}+\text { H.c., }
$$




$$
\begin{gathered}
g_{1} \frac{Y_{L}}{2} \bar{T}^{(1)} \gamma^{\mu} P_{L} t \gamma_{\mu}^{(1)}+\text { H.c., } \\
g_{2} \frac{1}{2} \bar{T}^{(1)} \gamma^{\mu} P_{L} t Z_{\mu}^{(1)}+\text { H.c., } \\
g_{2} \frac{1}{\sqrt{2}} \bar{B}^{(1)} \gamma^{\mu} P_{L} t W_{\mu}^{(1)-}+\text { H.c., }
\end{gathered}
$$

where $Y_{L / R}$ is the corresponding hypercharge, and $g_{1}$ and $g_{2}$ are the gauge coupling strengths of $U(1)_{Y}$ and $S U(2)_{W}$ interactions, respectively $\left(Y_{R}=4 / 3\right.$ and $\left.Y_{L}=1 / 3\right)$.
The $S U(2)_{W}$ doublet fields are defined as

$$
\begin{aligned}
q_{L} & =\left(\begin{array}{c}
T_{L} \\
B_{L}
\end{array}\right), \quad q^{(1)}=\left(\begin{array}{c}
T^{(1)} \\
B^{(1)}
\end{array}\right), \\
H & =\left(\begin{array}{c}
H^{+} \\
\frac{1}{\sqrt{2}}(v+h+i \chi)
\end{array}\right), \quad H^{(1)}=\left(\begin{array}{c}
H^{(1)+} \\
\frac{1}{\sqrt{2}}\left(h^{(1)}+i \chi^{(1)}\right)
\end{array}\right),
\end{aligned}
$$

where $f_{R / L}=P_{R / L} f$ for a fermion $f$.

The interactions involving the SM top quarks and KK Higgs are given by

$$
\begin{aligned}
\mathcal{L} & \ni \lambda_{t}\left[\bar{q}_{L} t_{R} i \sigma_{2} H^{*}+\bar{q}_{L} t_{R}^{(1)} i \sigma_{2} H^{(1) *}+\bar{q}_{L}^{(1)} t_{R} i \sigma_{2} H^{(1) *}\right]+\lambda_{b}\left[\bar{q}_{L} b_{R} H+\bar{q}_{L} b_{R}^{(1)} H^{(1)}+\bar{q}_{L}^{(1)} b_{R} H^{(1)}\right]+\text { H.c. } \\
= & \lambda_{t}\left[\bar{T}_{L} t_{R} \frac{1}{\sqrt{2}}(v+h)+\bar{T}_{L} t_{R}^{(1)} \frac{1}{\sqrt{2}}\left(h^{(1)}-i \chi^{(1)}\right)-\bar{B}_{L} t_{R}^{(1)} H^{(1)-}-\bar{B}_{L}^{(1)} t_{R} H^{(1)-}+\bar{T}_{L}^{(1)} t_{R} \frac{1}{\sqrt{2}}\left(h^{(1)}-i \chi^{(1)}\right)\right] \\
& +\lambda_{b}\left[\bar{B}_{L} b_{R} \frac{1}{\sqrt{2}}(v+h)+\bar{B}_{L} b_{R}^{(1)} \frac{1}{\sqrt{2}}\left(h^{(1)}+i \chi^{(1)}\right)+\bar{T}_{L} b_{R}^{(1)} H^{(1)+}+\bar{T}_{L}^{(1)} b_{R} H^{(1)+}+\bar{B}_{L}^{(1)} b_{R} \frac{1}{\sqrt{2}}\left(h^{(1)}+i \chi^{(1)}\right)\right]+\text { H.c. }
\end{aligned}
$$

where $m_{t(b)}=\frac{\lambda_{t(b)} v}{\sqrt{2}}$, and $v=\frac{2 m_{W}}{g_{2}} \approx 246 \mathrm{GeV}$ are the top (bottom) quark mass and the Higgs vacuum expectation value, respectively.

\section{ANALYSIS}

We consider the monotop signature arising from (i) the third generation extradimensional model $p p \rightarrow t \mathrm{KK}_{f}^{(1)} \mathrm{KK}_{b}^{(1)}$ and (ii) the minimal top-partner scenario $p p \rightarrow t t^{(1)} h^{(1)}$, where $\mathrm{KK}_{f}^{(1)}$ represents any third generation KK quark $t^{(1)}$, $T^{(1)}$ or $B^{(1)}$, and $\mathrm{KK}_{b}^{(1)}$ any $\mathrm{KK}$ boson that couples to the top quark, as illustrated in Fig. $1 .^{2}$ In scenario (i), as illustrated in the figure, many different processes effectively contribute to the same monotop $+E_{T}$ final state, as all KK particles are quasimass degenerate, being essentially invisible with soft and undetected decay products. Following Refs. [1,2], we set in our analysis $m_{\gamma^{(1)}}=m_{Z^{(1)}}=m_{\chi^{(1)}}=m_{h^{(1)}}, \quad m_{H^{(1) \pm}}=m_{W^{(1) \pm}}=m_{\gamma^{(1)}}+$ $1 \mathrm{GeV}, m_{t^{(1)}}=m_{T^{(1)}}=m_{B^{(1)}}=m_{\gamma^{(1)}}+8 \mathrm{GeV}$, and assume no particle has a detector scale lifetime (except for the stable lightest KK particles).

In this analysis, we concentrate on the leptonic monotop signature; see Fig. 2 for a schematic monotop event display. This channel is characterized by the presence of an isolated lepton $\ell=e, \mu$, one $b$-tagged jet, and missing energy $\mathbb{E}_{T}$. The dominant backgrounds for this signature are $\bar{t} t+$ jets, $t W, t Z$, and $W \bar{b} b$ production processes.

\footnotetext{
${ }^{2}$ The KK gluon is not included here, since it is often the heaviest particle in UED models.
}

In our analysis, we generate the $\bar{t} t+$ jets sample with ALPGEN+Pythia6 [59] merged up to one jet, with the MLM multijet merging algorithm. The signal and additional background samples are generated with MADGRAPH5_AMC@NLO+PythiA8 [60,61], accounting for hadronization and underlying event effects. Detector effects are simulated with the DELPHES3 package [62]. Higher order corrections are accounted for by normalizing the total $\bar{t} t$ rate to the NNLO + NNLL cross section $(831 \mathrm{pb}$ [63]), and the $t W$ and $t Z$ to their next-to-leading order predictions (71 $\mathrm{pb}$ [64]) and (0.88 pb [65]), respectively. ${ }^{3}$ We use the K-factor of 1.5 for the signal processes.

We start our analysis requiring one isolated lepton $p_{T \ell}>$ $10 \mathrm{GeV}$ and $\left|\eta_{\ell}\right|<2$.4. Jets are defined via the anti- $k_{T}$ jet algorithm $R=0.4, p_{T j}>20 \mathrm{GeV}$ and $\left|\eta_{j}\right|<2.5$ with the FASTJET package [66]. We require one $b$-jet with $b$-tagging efficiency of $70 \%$ that is associated to a mistag rate of $15 \%$ for $c$-quarks and $1 \%$ for light quarks [67]. To tame the $\bar{t} t$ and $W \bar{b} b$ backgrounds, we explore the Jacobian peak structure for the signal, imposing $m_{b \ell}<150 \mathrm{GeV}$. The $W \bar{b} b$ background does not present this shape since it does not have a top quark in the event and the $\bar{t} t$ typically produce a large tail, coming from events with the $b$ and $l$ combination from different top-quark decays.

We further control the background with the transverse mass $m_{T}=\sqrt{2 p_{T \ell} E_{T}^{\dagger}\left(1-\cos \phi_{\ell, E_{T}^{\prime}}\right)}$, requiring

\footnotetext{
${ }^{3}$ The literature does not provide higher order corrections to the signal under consideration. We indicate the importance of its determination for future studies.
} 


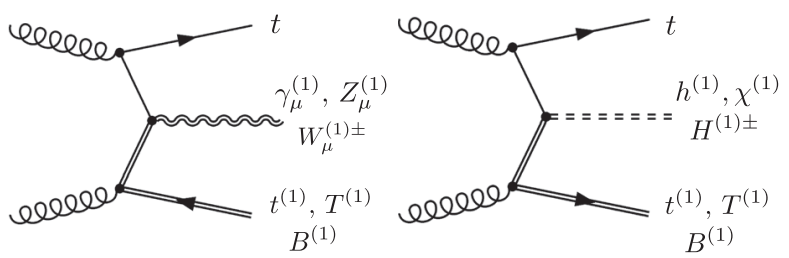

FIG. 1. Representative set of Feynman diagrams resulting in the monotop signature in the third generation extradimensional model.

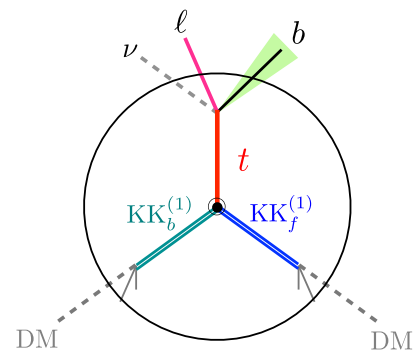

FIG. 2. Schematic monotop event display. The grey dashed lines represent invisible particles, whereas the thin grey lines depict soft particles that do not pass the minimum selection criteria.

$m_{T}>100 \mathrm{GeV}$ that explores the sharp drop above the $m_{T} \sim m_{W}$ for the semileptonic $\bar{t} t$ and $W \bar{b} b$ samples.

In Fig. 3, we present the resulting missing energy distributions for signal and background for the third generation extradimensional model and the corresponding SUSY case. We set the top/bottom partner masses $m_{t^{(1)}}=m_{T^{(1)}}=m_{B^{(1)}}=317 \mathrm{GeV}$. The signal distribution exhibits a lower suppression with $\mathbb{E}_{T}$ compared to the

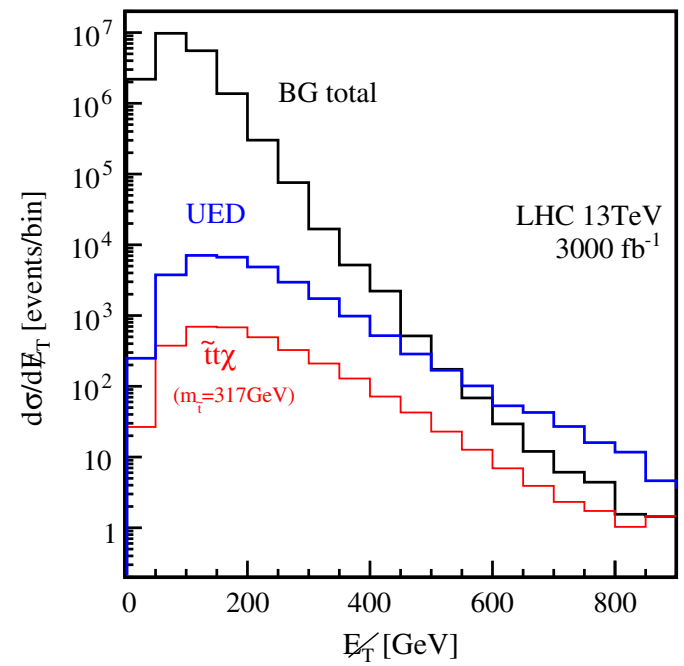

FIG. 3. The missing energy $\mathbb{E}_{T}$ distributions for the SM background (black) and the monotop signal in the third generation extradimensional model [case (i), blue]. The distribution for the SUSY case with the $\tilde{t} t \tilde{\chi}_{1}^{0}$ channel is also shown in red. For illustration purposes, we show the SUSY and UED distributions with the same top-partner masses. We assume the $13 \mathrm{TeV}$ LHC with $3 \mathrm{ab}^{-1}$ of integrated luminosity. backgrounds. We exploit this fact and define three signal regions with different requirements on the missing energy threshold, $E_{T} / \mathrm{GeV}>550,600$, and 650 . The detailed signal and background cut-flow is displayed in Table I. For scenario (i), the main contribution comes from $B^{(1)} W_{\mu}^{(1) \pm} t$ subchannel accounting for $28 \%$ of the total rate, followed by $B^{(1)} H^{(1) \pm} t$ with $16 \%, T^{(1)} Z_{\mu}^{(1)} t$ with $14 \%, T^{(1)} h^{(1)} t$ with $8.8 \%, t^{(1)} \gamma_{\mu}^{(1)} t$ with $8.7 \%, T^{(1)} \chi^{(1)} t$ with $8.2 \%, t^{(1)} h^{(1)} t$ with $8.0 \%, t^{(1)} \chi^{(1)} t$ with $7.9 \%$ and $T^{(1)} \gamma_{\mu}^{(1)} t$ with $0.5 \%$. In summary, $B^{(1)}, T^{(1)}, t^{(1)}$ involved process, contributes $43 \%, 32 \%, 25 \%$, respectively. These fractions are understood straightforwardly from the gauge couplings $g_{1}, g_{2}$ and top Yukawa coupling $\lambda_{t}$ and hypercharges. Here we ignore the processes whose amplitudes are proportional to the bottom Yukawa coupling $\lambda_{b}$, as they present a subleading contribution.

In Fig. 4, we show $\mathcal{S} / \sqrt{\mathcal{B}}$ (solid lines), $\mathcal{S} / \mathcal{B}$ (dashed lines), $\mathcal{S}$ (dotted lines) as functions of the top-partner mass $m_{t^{(1)}}$ for scenario (i), assuming the mass splitting $m_{t^{(1)}}-$ $m_{X^{(1)}}=8 \mathrm{GeV}$ and the $13 \mathrm{TeV}$ LHC with $\int \mathcal{L} d t=3 \mathrm{ab}^{-1}$. For completeness, we show the results for our different signal regions, $\mathbb{E}_{T} / \mathrm{GeV}>550$ (blue), 600 (green), and 650 (orange). They provide very similar sensitivities in $\mathcal{S} / \sqrt{\mathcal{B}}$. One can see that the top-partner mass can be probed up to $m_{t^{(1)}} \sim 630 \mathrm{GeV}$ at $95 \%$ C.L. in scenario (i). A higher $\mathbb{E}_{T}$ cut predicts smaller number of events but $\mathcal{S} \gtrsim 10$ can still be achieved with $3 \mathrm{ab}^{-1}$ around $m_{t^{(1)}} \sim 630 \mathrm{GeV}$, while keeping $\mathcal{S} / \mathcal{B} \gtrsim 0.3$.

For the minimal top-partner simplified scenario (ii), the sensitivity can be estimated by rescaling the cross section according to the contribution of the subprocesses quoted above, as we have checked that the missing energy distributions for all relevant processes are practically identical. Since scenario (ii) amounts to a signal that is purely $t^{(1)} h^{(1)} t$, its rate is tantamount to only $8 \%$ of the total rate in scenario (i), as mentioned above. We find that the sensitivity reaches only just below $300 \mathrm{GeV}$ in scenario (ii). If we assume the signal strength of 2 , corresponding for instance to an inclusion of the $t^{(1)} \chi^{(1)} t$ process, we find the $95 \% \mathrm{CL}$ expected limit on the top-partner mass $m_{t^{(1)}} \gtrsim 400 \mathrm{GeV}$, which should be directly compared with the sensitivity to the stop mass, $m_{\tilde{t}} \gtrsim 380 \mathrm{GeV}$, in the natural SUSY scenario where two different channels corresponding to the two degenerate Higgsino-like neutralinos $\left(\tilde{\chi}_{1}^{0}, \tilde{\chi}_{2}^{0}\right)$ contribute.

If the DM is selected to the spin- $1 \mathrm{KK}$ photon $\gamma_{\mu}^{(1)}$ instead of the KK Higgs $h^{(1)}$ in the minimal scenario, the strength of interaction is replaced by the $U(1)_{Y}$ gauge coupling multiplied by the hypercharge $Y_{R}=4 / 3$. As $\gamma_{\mu}^{(1)}$ has a larger degree of freedom compared to $h^{(1)}$, the cross section of the $p p \rightarrow t^{(1)} \gamma_{\mu}^{(1)} t$ process appears to be almost identical to that of $p p \rightarrow t^{(1)} h^{(1)} t$. We therefore have a very similar conclusion for the $\left(t^{(1)}, \gamma_{\mu}^{(1)}\right)$ minimal simplified scenario. 


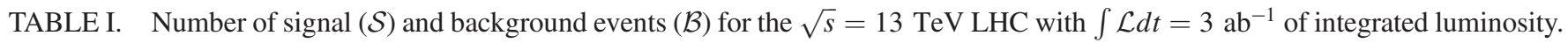
We display the signal results for two benchmark points: $\left(m_{t^{(1)}}, m_{X^{(1)}}\right) / \mathrm{GeV}=(492,484)$ and $(697609)$, where $X^{(1)} \equiv$ $\gamma^{(1)}, Z^{(1)}, W^{(1)}, h^{(1)}, \chi^{(1)}, H^{(1) \pm}$. We show the signal sensitivity in brackets $(\mathcal{S} / \sqrt{\mathcal{B}}, \mathcal{S} / \mathcal{B})$ in the last three columns. We consider all $t^{(1)}, T^{(1)}, B^{(1)}$ related modes.

\begin{tabular}{|c|c|c|c|c|c|c|c|}
\hline Process & $\sigma$ & Baseline & $m_{b \ell}<150$ & $m_{T}>100$ & $E_{T}>550$ & $E_{T}>600$ & $E_{T}>650$ \\
\hline$t \bar{t}$ & $831 \mathrm{pb}$ & $206 \times 10^{6}$ & $165 \times 10^{6}$ & $17.7 \times 10^{6}$ & 55.2 & 25.0 & 11.2 \\
\hline$t W$ & $71 \mathrm{pb}$ & $26.2 \times 10^{6}$ & $20.7 \times 10^{6}$ & $1.68 \times 10^{6}$ & 55.5 & 24.3 & 10.4 \\
\hline$t Z$ & $0.88 \mathrm{pb}$ & $22.8 \times 10^{3}$ & $21.6 \times 10^{3}$ & $7.3 \times 10^{3}$ & 8.0 & 4.9 & 3.5 \\
\hline$W b \bar{b}$ & $7.65 \mathrm{pb}$ & $1.82 \times 10^{6}$ & $1.51 \times 10^{6}$ & $42.3 \times 10^{3}$ & 1.4 & 0.7 & 0.3 \\
\hline background $(\mathrm{BG})$ total & $903 \mathrm{pb}$ & $226 \times 10^{6}$ & $41.1 \times 10^{6}$ & $19.4 \times 10^{6}$ & 120.1 & 54.9 & 25.5 \\
\hline benchmark point (BP) (317 309) & $269 \mathrm{fb}$ & 47996 & 45133 & 29750 & $\begin{array}{c}195.1 \\
(17.8,1.6)\end{array}$ & $\begin{array}{c}131.2 \\
(17.7,2.4)\end{array}$ & $\begin{array}{c}92.0 \\
(18.3,3.6)\end{array}$ \\
\hline BP (492 484) & $32.7 \mathrm{fb}$ & 5502 & 5131 & 3529 & $\begin{array}{c}57.9 \\
(5.3,0.48)\end{array}$ & $\begin{array}{c}38.0 \\
(5.1,0.69)\end{array}$ & $\begin{array}{c}24.6 \\
(4.9,0.96)\end{array}$ \\
\hline BP (617 609) & $9.56 \mathrm{fb}$ & 1588 & 1471 & 1048 & $\begin{array}{c}22.8 \\
(2.1,0.19)\end{array}$ & $\begin{array}{c}15.1 \\
(2.0,0.28)\end{array}$ & $\begin{array}{c}11.4 \\
(2.2,0.44)\end{array}$ \\
\hline
\end{tabular}

Finally, as in the SUSY scenario, monojet searches can provide important constraints for the degenerate spectrum. We repeat similar analysis performed in Ref. [42] with the KK tops $\left(t^{(1)}, T^{(1)}\right)$ and KK bottom $\left(B^{(1)}\right)$ only. Since the authors of Ref. [42] include the KK gluon and all three generations of KK quarks in their monojet study, their limit does not apply directly to our case, where only $t^{(1)}, T^{(1)}$, $B^{(1)}$ are considered. We show the current bound on the KK top mass in Fig. 5, using the data corresponding to an integrated luminosity of $36.1 \mathrm{fb}^{-1}$ at the $13 \mathrm{TeV}$ LHC. We used the model-independent $95 \%$ C.L. upper limits on signal cross section in the final state with an energetic jet and large missing transverse momentum reported by

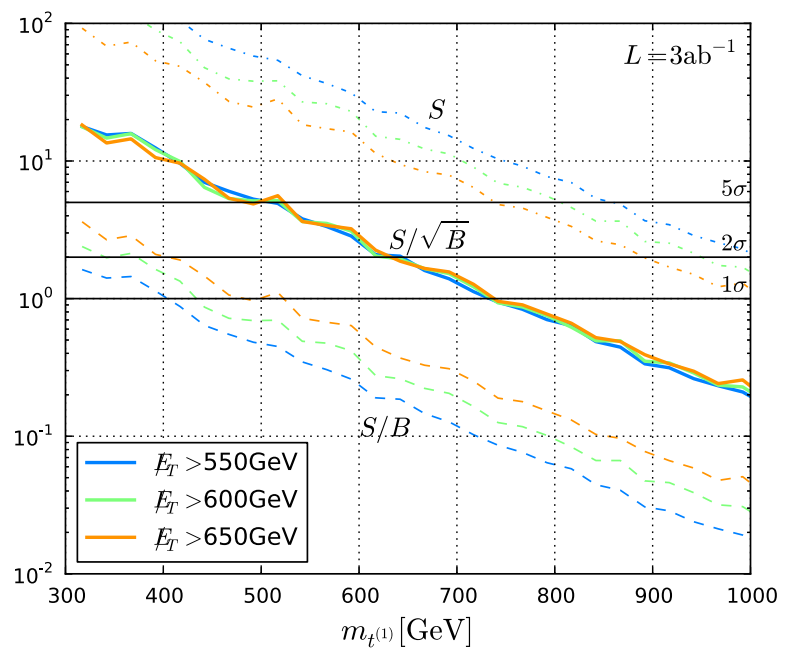

FIG. 4. Sensitivity lines $\mathcal{S} / \sqrt{\mathcal{B}}$ (solid) and $\mathcal{S} / \mathcal{B}$ (dashed) with $\int \mathcal{L} d t=3 \mathrm{ab}^{-1}$ as functions of the top-partner mass $m_{t^{(1)}}$ in the third generation extradimensional model. The mass splitting $m_{t^{(1)}}-m_{X^{(1)}}=8 \mathrm{GeV}$ is assumed. The results for different missing energy selections, $\mathbb{E}_{T} / \mathrm{GeV}>550$ (blue), 600 (green), and 650 (orange), are shown.
ATLAS [68]. Our analysis indicates that the current monojet study excludes the $\mathrm{KK}$ top mass up to $\sim 750 \mathrm{GeV}$, which corresponds to $\sim 1.6 \mathrm{TeV}$ for a higher luminosity of $3 \mathrm{ab}^{-1}$ via a rough rescaling based on [69]. This is more powerful than the sensitivity of the monotop channel, which implies that the monotop channel is not a discovery channel and we should expect excesses both in the monojet and monotop channels if we have a light top partner in the spectrum. Significant improvements in the monotop sensitivity can be obtained by also exploiting the hadronic monotop final state [2].

It has been pointed out that the monotop channel has a complementary role in the monojet channel [1]. The final state of the monojet channel only contains the jets originated from the QCD initial state radiation. It therefore does not carry the information on the details of the top-partner and DM sectors. Conversely, the existence of the top quark

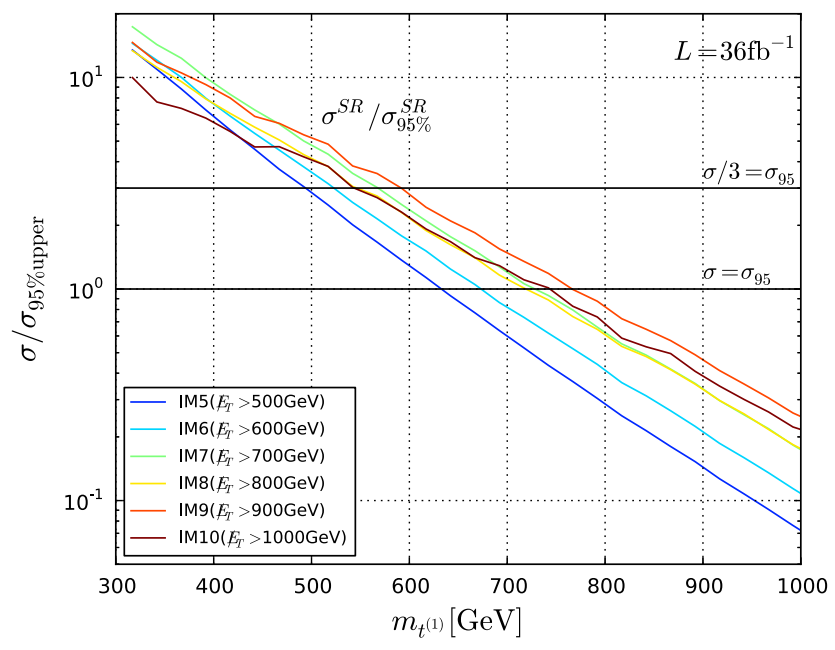

FIG. 5. Current bound on the mass of the fermionic top partner from the monojet study at $95 \%$ C.L [68]. We display the results for different missing energy selections. 
in the monotop channel is a clear indication that the process is related to the third generation. The helicity of the top quark can also be measured by looking at the angular distribution between the charged lepton and the $b$-quark in the final state [1], which provides important information on the chirality structure of the top partner. Moreover, unlike the monojet channel, the production rate depends not only on the QCD coupling but also on the couplings of new interactions involving the top quark and the top partner. For example, in the simplified scenario (ii), one can introduce the scaling factor $\xi$ as

$$
\mathcal{L} \ni \xi \frac{\lambda_{t}}{\sqrt{2}} \bar{T}_{L} t_{R}^{(1)} h^{(1)} .
$$

With this parametrization, the signal strength scales as $\xi^{2}$ and one can set the limit on $\xi$ using the monotop channel. Using the previous analysis, we have estimated the current and projected sensitivities on $\xi$ for $\int \mathcal{L} d t=36,300$, and $3000 \mathrm{fb}^{-1}$ presented in Fig. 6. One can see that for, e.g., $m_{t^{(1)}} \simeq 800 \mathrm{GeV}$ the high luminosity LHC can prove $\xi$ up to around 6 . The $\xi$ can also be effectively increased by introducing additional particles that couple to the top quark and the top partner (bottom partner) in the same way as, e.g., $\gamma_{\mu}^{(1)}$ and $h^{(1)}\left(W_{\mu}^{(1)}\right.$ and $\left.H^{(1) \pm}\right)$. If those new particles are only electroweakly charged, the enhancement of $\xi$ will be independent of the monojet channel. On the other hand, if they are colored, such as the KK gluon, though the monotop channel is significantly enhanced (due to, e.g., $p p \rightarrow t t^{(1)} g^{(1)}$ for the KK gluon case), the rate of monojet channel also increases due to the pair production of those colored particles. Even though the sensitivity of the monotop channel is in general weaker than that of the monojet channel, it is important to look for the monotop channel, since this process provides important information on the

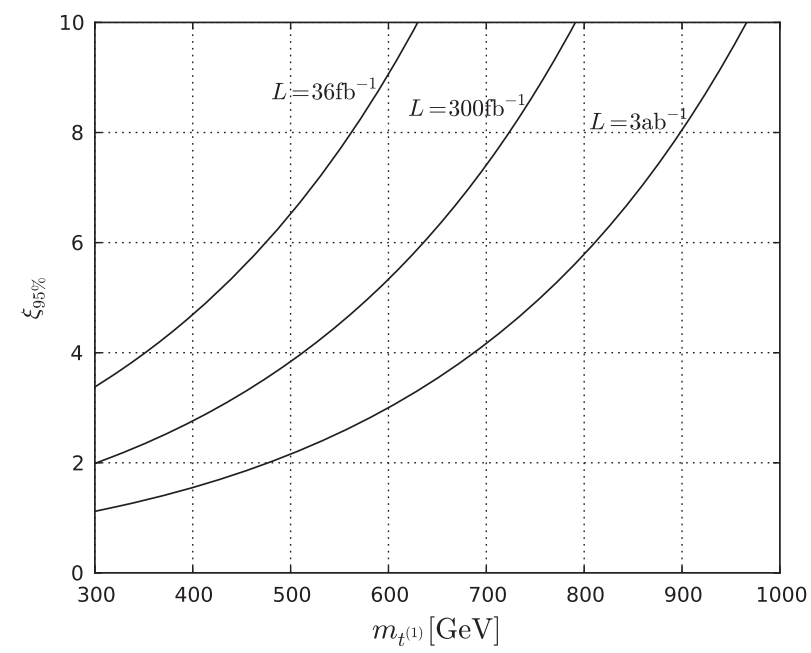

FIG. 6. The current and projected sensitivities on $\xi$ [introduced in Eq. (8)]. The integrated luminosity of 36300 , and $3000 \mathrm{fb}^{-1}$ is considered. top partner and DM sectors in the fermionic top-partner models.

\section{CONCLUSION}

The prospects of observing the monotop signatures at the LHC arising from fermionic top-partner models have been studied. Such a signature was previously studied in the $p p \rightarrow \tilde{t} t \tilde{h}^{0}$ process in the context of Natural Supersymmetry, where the stop and Higgsino present a very small mass gap, leading to soft and thus undetectable decay products from $\tilde{t}$ decays [1,2]. Interestingly, a similar setup arises in the UED framework, where the compressed mass spectra are naturally expected. In extradimensional models many different channels contribute to the same monotop final state, resulting in a large gain in the signal rate and the sensitivity. We showed that the monotop channel can explore the toppartner masses up to $630 \mathrm{GeV}$ (or $300 \mathrm{GeV}$ in the simplified scenario) at the high luminosity LHC. Possible improvements in this bound can be obtained by taking also the hadronic monotop channel into account. We have compared the monojet and monotop channels and found that the sensitivity of the monojet channel is in general superior to the monotop channel. We have also argued that despite weaker sensitivity of the monotop channel, it is important to observe and investigate this process since it allows us to access the information on the fermionic top partners and the new particles that couple to the top quark and the top partner. Since this channel has not been investigated experimentally in the contexts of supersymmetry and extradimensional models, we hope that more detailed studies will be performed by the experimental collaborations at the LHC.

\section{ACKNOWLEDGMENTS}

K. S. thanks the TU Munich for hospitality during the final stages of this work and has been partially supported by the German Research Foundation (DFG) cluster of excellence EXC 153 "Origin and Structure of the Universe," by the Collaborative Research Center Grant No. SFB1258. D. G. was funded by U.S. National Science Foundation under Grant No. PHY-1519175. K. K. is supported in part by U.S. Department of Energy under Award No. DE-SC0017965 and in part by the University of Kansas General Research Fund Grant No. 2151081. The work of K. S. is partially supported by the National Science Centre, Poland, under Grants No. DEC-2014/15/B/ST2/ 02157, No. DEC-2015/18/M/ST2/00054, and No. 2015/19/ D/ST2/03136. M. T. is supported in part by the Japan Society for the Promotion of Science Grant-in-Aid for Scientific Research Grants No. JP16H03991, No. JP16H02176, and No. 17H05399, and by World Premier International Research Center Initiative (WPI Initiative), MEXT, Japan. 
[1] D. Goncalves, K. Sakurai, and M. Takeuchi, Phys. Rev. D 94, 075009 (2016).

[2] D. Goncalves, K. Sakurai, and M. Takeuchi, Phys. Rev. D 95, 015030 (2017).

[3] B. Fuks, P. Richardson, and A. Wilcock, Eur. Phys. J. C 75, 308 (2015).

[4] G. H. Duan, K.-i. Hikasa, L. Wu, J. M. Yang, and M. Zhang, J. High Energy Phys. 03 (2017) 091.

[5] K.-i. Hikasa, J. Li, L. Wu, and J. M. Yang, Phys. Rev. D 93, 035003 (2016).

[6] G. Aad et al. (ATLAS Collaboration), Eur. Phys. J. C 75, 79 (2015).

[7] V. Khachatryan et al. (CMS Collaboration), Phys. Rev. Lett. 114, 101801 (2015).

[8] H. Davoudiasl, D. E. Morrissey, K. Sigurdson, and S. Tulin, Phys. Rev. D 84, 096008 (2011).

[9] J. F. Kamenik and J. Zupan, Phys. Rev. D 84, 111502 (2011).

[10] J. Andrea, B. Fuks, and F. Maltoni, Phys. Rev. D 84, 074025 (2011).

[11] E. Alvarez, E. C. Leskow, J. Drobnak, and J. F. Kamenik, Phys. Rev. D 89, 014016 (2014).

[12] J.-L. Agram, J. Andrea, M. Buttignol, E. Conte, and B. Fuks, Phys. Rev. D 89, 014028 (2014).

[13] I. Boucheneb, G. Cacciapaglia, A. Deandrea, and B. Fuks, J. High Energy Phys. 01 (2015) 017.

[14] J. Wang, C. S. Li, D. Y. Shao, and H. Zhang, Phys. Rev. D 86, 034008 (2012).

[15] M. Aaboud et al. (ATLAS Collaboration), Phys. Rev. D 94, 032005 (2016).

[16] V. Khachatryan et al. (CMS Collaboration), Phys. Lett. B 767, 403 (2017).

[17] R. Kitano and Y. Nomura, Phys. Rev. D 73, 095004 (2006).

[18] M. Papucci, J. T. Ruderman, and A. Weiler, J. High Energy Phys. 09 (2012) 035.

[19] L. J. Hall, D. Pinner, and J. T. Ruderman, J. High Energy Phys. 04 (2012) 131.

[20] N. Desai and B. Mukhopadhyaya, J. High Energy Phys. 05 (2012) 057.

[21] K. Ishiwata, N. Nagata, and N. Yokozaki, Phys. Lett. B 710, 145 (2012).

[22] K. Sakurai and K. Takayama, J. High Energy Phys. 12 (2011) 063.

[23] S.-G. Kim, N. Maekawa, K. I. Nagao, M. M. Nojiri, and K. Sakurai, J. High Energy Phys. 10 (2009) 005.

[24] C. Wymant, Phys. Rev. D 86, 115023 (2012).

[25] H. Baer, V. Barger, P. Huang, A. Mustafayev, and X. Tata, Phys. Rev. Lett. 109, 161802 (2012).

[26] L. Randall and M. Reece, J. High Energy Phys. 08 (2013) 088.

[27] J. Cao, C. Han, L. Wu, J. M. Yang, and Y. Zhang, J. High Energy Phys. 11 (2012) 039.

[28] M. Asano and T. Higaki, Phys. Rev. D 86, 035020 (2012).

[29] H. Baer, V. Barger, P. Huang, and X. Tata, J. High Energy Phys. 05 (2012) 109.

[30] J. A. Evans, Y. Kats, D. Shih, and M. J. Strassler, J. High Energy Phys. 07 (2014) 101.

[31] E. Hardy, J. High Energy Phys. 10 (2013) 133.

[32] G. D. Kribs, A. Martin, and A. Menon, Phys. Rev. D 88, 035025 (2013).
[33] B. Bhattacherjee, J. L. Evans, M. Ibe, S. Matsumoto, and T. T. Yanagida, Phys. Rev. D 87, 115002 (2013).

[34] K. Rolbiecki and K. Sakurai, J. High Energy Phys. 09 (2013) 0041.

[35] M. Papucci, K. Sakurai, A. Weiler, and L. Zeune, Eur. Phys. J. C 74, 3163 (2014).

[36] T. Appelquist, H.-C. Cheng, and B. A. Dobrescu, Phys. Rev. D 64, 035002 (2001).

[37] H. Georgi, A. K. Grant, and G. Hailu, Phys. Rev. D 63, 064027 (2001).

[38] H.-C. Cheng, K. T. Matchev, and M. Schmaltz, Phys. Rev. D 66, 056006 (2002).

[39] H.-C. Cheng, K. T. Matchev, and M. Schmaltz, Phys. Rev. D 66, 036005 (2002).

[40] T. Flacke, K. Kong, and S. C. Park, J. High Energy Phys. 05 (2013) 111.

[41] T. Flacke, D. W. Kang, K. Kong, G. Mohlabeng, and S. C. Park, J. High Energy Phys. 04 (2017) 041.

[42] D. Choudhury and K. Ghosh, Phys. Lett. B 763, 155 (2016).

[43] N. Deutschmann, T. Flacke, and J. S. Kim, Phys. Lett. B 771, 515 (2017).

[44] J. Beuria, A. Datta, D. Debnath, and K. T. Matchev, arXiv:1702.00413.

[45] G. Belanger, M. Kakizaki, and A. Pukhov, J. Cosmol. Astropart. Phys. 02 (2011) 009.

[46] G. Servant and T. M. P. Tait, New J. Phys. 4, 99 (2002).

[47] K. Kong and K. T. Matchev, J. High Energy Phys. 01 (2006) 038.

[48] H.-C. Cheng, J. L. Feng, and K. T. Matchev, Phys. Rev. Lett. 89, 211301 (2002).

[49] S. Arrenberg, L. Baudis, K. Kong, K. T. Matchev, and J. Yoo, Phys. Rev. D 78, 056002 (2008).

[50] A. Datta, K. Kong, and K. T. Matchev, Phys. Rev. D 72, 096006 (2005); 72, 119901(E) (2005).

[51] S. Baek, P. Ko, and P. Wu, J. High Energy Phys. 10 (2016) 117.

[52] CERN Collaboration, Report No. ATLAS-CONF-2017022, Geneva, 2017.

[53] CERN Collaboration, Report No. ATLAS-CONF-2017060, Geneva, 2017.

[54] A. Datta and A. Shaw (Indian Association for the Cultivation of Science), Phys. Rev. D 95, 015033 (2017).

[55] A. Biswas, S. K. Patra, and A. Shaw, arXiv:1708.08938.

[56] T. Flacke, A. Menon, and D. J. Phalen, Phys. Rev. D 79, 056009 (2009).

[57] Y. Ishigure, M. Kakizaki, and A. Santa, arXiv:1611.06760.

[58] G.-Y. Huang, K. Kong, and S. C. Park, J. High Energy Phys. 06 (2012) 099.

[59] M. L. Mangano, M. Moretti, F. Piccinini, R. Pittau, and A. D. Polosa, J. High Energy Phys. 07 (2003) 001.

[60] J. Alwall, R. Frederix, S. Frixione, V. Hirschi, F. Maltoni, O. Mattelaer, H. S. Shao, T. Stelzer, P. Torrielli, and M. Zaro, J. High Energy Phys. 07 (2014) 079.

[61] T. Sjostrand, S. Mrenna, and P. Z. Skands, Comput. Phys. Commun. 178, 852 (2008).

[62] S. Ovyn, X. Rouby, and V. Lemaitre, arXiv:0903.2225 [Comput. Phys. Commun. (to be published)].

[63] M. Czakon, P. Fiedler, and A. Mitov, Phys. Rev. Lett. 110, 252004 (2013). 
[64] LHC Top Working Group, https://twiki.cern.ch/twiki/bin/ view/LHCPhysics/SingleTopRefXsec, 2016.

[65] J. Campbell, R. K. Ellis, and R. Rontsch, Phys. Rev. D 87, 114006 (2013).

[66] M. Cacciari, G. P. Salam, and G. Soyez, Eur. Phys. J. C 72, 1896 (2012).
[67] CMS Collaboration, Report No. CMS-PAS-BTV-13-001, 2013.

[68] ATLAS Collaboration, Report No. ATLAS-CONF-2017060, 2017.

[69] C. Reach, $(\beta)$, http://collider-reach.web.cern.ch, 2014. 